\title{
Fractionated Repolarization Induced by Sotalol in Healthy Subjects
}

\author{
M Vaglio, JP Couderc, X Xia, W Zareba
}

\author{
Heart Research Follow-up-Program, University of Rochester Medical Center, Rochester, NY, USA
}

\begin{abstract}
Over the past five years, regulatory authorities have been increasingly concerned with $Q T$ prolongations induced by non-cardiac drug and have recommended pharmaceutical companies to include a careful assessment of the QT interval in their drug development programs.

There are controversies around the predictive value of $Q T$ prolongation in safety-drug assessment studies. The prolongation of the QT interval is an imperfect, but accepted, surrogate marker of drug cardiac toxicity. In this study, we hypothesize that the inhomogeneous effect of $Q T$ prolonging drug in the different layers of the myocardium would not only delay cardiac repolarization, but also perturb the repolarization wavefront on surface ECGs.

Principal component analysis (PCA) was applied to the 12-lead ECG Holter recordings. The first two eigenvectors $\left(e v_{1}, e v_{2}\right)$ were computed. From PCA, several parameters were calculated based on the first eigenvector and on the T-loop. We demonstrated slower repolarization and perturbed T-wave front; 30 min after sotalol administration, turbulence of repolarization velocity increased by $9.02 \%$, $p<0.05$, occurring prior to QT prolongation. These abnormalities were mainly located in the ascending part of the T-wave, whereas the descending part seemed to be less affected at this early phase.

In conclusion, analyzing repolarization morphology might help identifying early drug-induced repolarization changes, which could be missed when relying exclusively on QT measurements.
\end{abstract}

\section{Introduction}

Sotalol is a QT-prolonging drug that has been associated with Torsades de Pointes, a life threatening form of ventricular arrythmia. This drug delays mainly the rapidly $\left(I_{K r}\right)$ delayed rectifier potassium currents of the myocardial cells prolonging their action potentials. The resulting effect on the surface ECG is an increased QT interval duration.

The QT is a challenging interval to measure since the QT interval determination relies on the identification of the end of the T-wave, which might be visually assessed differently according to the experience and training of the cardiologist/technician [1]. There is a need for identifying more robust and more sensitive methods reflecting effect of drugs on repolarization.

In this study, we hypothesize that the prolonging effect of sotalol in different layers of the myocardium may alter the conduction velocity of the ventricular repolarization phase. The aim of this study was to measure sotalol-induced repolarization abnormalities of the T-wave and the T-loop morphology in surface ECGs recorded using 12-lead Holter system.

\section{Methods}

\subsection{Study population}

Twenty-four hours 12-lead ECG Holter were recorded in 38 healthy subjects on two consecutive days. There were 11 females and 27 males. No drug was given on the first day (baseline), while $160 \mathrm{mg}$ of sotalol (Betapace ${ }^{\mathrm{B}}$ ) was given on the second day (on drug) one hour after the start of the recording. Subjects were at rest in supine position. Recordings were done at the same time each day for each patient. Sotalol blood plasma concentration was measured with each ECG. The protocol is described in details elsewhere [2].

\subsection{ECG recordings}

Twelve-lead Holter equipment (Mortara Instrument, Milwaukee, WI) was used to record 24-hour Holter digital ECG signal at a sampling frequency of $180 \mathrm{~Hz}$ and with 16 bit amplitude resolution $(2.5 \mu \mathrm{V})$.

Eight leads were recorded and the remaining leads (aVR, aVL, aVF and lead III) were computed. The PCA analysis was applied to the eight original leads.

\subsection{Measurement technique}

Measurements are based on median beats from 10 consecutive cardiac cycles throughout entire Holter recording. 
Only beats with stable heart rate (HR) are taken into account $(90 \% \overline{H R}<H R<110 \% \overline{H R})$. PCA was applied on the ECG signals, starting at the J point and ending $220 \mathrm{~ms}$ before the next $\mathrm{R}$ peak to ensure the analysis encompassed all components of the ventricular repolarization process from the surface ECGs.

The PCA is a statistical method for reducing the dimensions of a system while minimizing the loss of information [3]. The new variables describing the ECG signals are linear combinations of the ECG leads. In electrocardiography, where there is correlation between different leads, PCA of the repolarization segment has been previously used for the quantification of ventricular heterogeneity [4], [5], [6] and [7].

It relies on the computation of the Singular Value Decomposition (SVD) [8], in which any matrix $\mathbf{A}(M x N)$ can be written as:

$$
\mathbf{A}=\mathbf{U S V}^{\mathbf{T}}
$$

where $\mathbf{U}$ is a $(M \times N)$ column-orthogonal matrix, $\mathbf{S}$ a diagonal matrix with positive or zero elements (the singular values), and $\mathbf{V}$ an $(N \times N)$ orthogonal matrix containing the right singular vectors.

Being $\mathbf{A}$ a matrix ( $M x 8$ ), having in columns the 8 leads and in row the $M$ samples of the repolarization signal of one cardiac beat, applying SVD, we get $\mathbf{U}, \mathbf{S}$ and $\mathbf{V}^{\mathbf{T}}$ matrices; $\mathbf{S}$ will contain the 8 eigenvalues in descending order. Multiplying $\mathbf{A} \cdot \mathbf{V}$, provides the projection of our original data, on the principal components.

The first two components $\left(e v_{1}, e v_{2}\right)$ are the eigenleads, while the plane $\left(e v_{1} \perp e v_{2}\right)$ is the preferential plane (Fig. $1)$.

\subsection{Investigated repolarization parame- ters}

In our study, we measured the QT interval from lead II using our own developed software for Comprehensive Analysis of the repolarization Signal (COMPAS) that provides repolarization measurements [9]. PCA measurement were obtained from the COMPAS PCA analysis package.

The T-wave morphology was measured using the following quantifiers, computed on the first eigenlead:

- LST, left slope of T-wave on $e v_{1}$, defined as the maximum slope of the ascending part of the T-wave, indicating the maximum velocity of the early repolarization process; - TRV, turbulence of repolarization velocity, defined as the number of deflections within the first derivative of the $\mathrm{J}$ - T-Apex interval on $e v_{1}$. This index measures the presence of notches and perturbed repolarization signal.

Other parameter designed from PCA looked at TLoop morphology, on the preferential plane (identified by $\left.e v_{1} \perp e v_{2}\right)$, see Fig. 1:
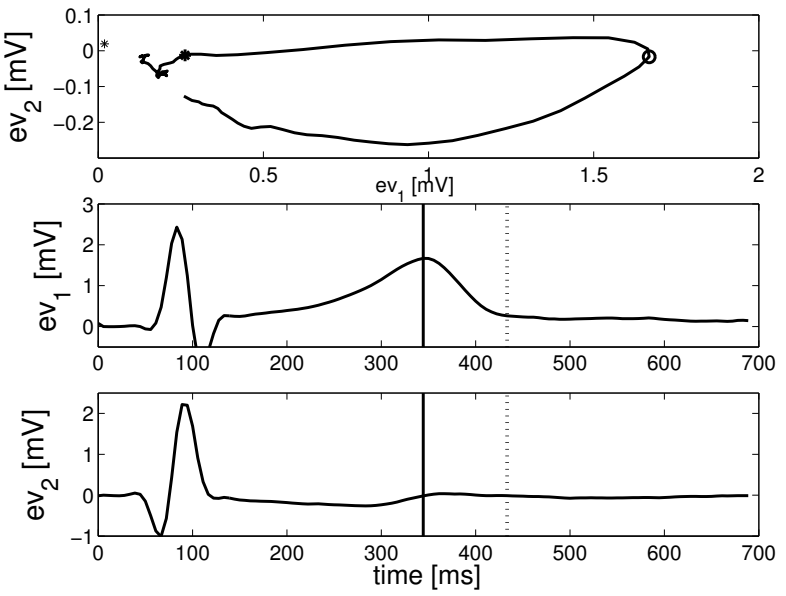

Figure 1. From top to bottom: T-loop in the preferential plane $\left(e v_{1} \perp e v_{2}\right), e v_{1}, e v_{2}$. MV and T-wave end are identified using solid and dotted markers, respectively.

- Maximum vector (MV), maximum amplitude of T-loop from the projection of beginning of QRS, on preferential plane, see Fig. 1 and 2;

- QT interval calculated on T-Loop $\left(Q T_{e}\right)$, where the end of the T-wave is identified on the point $T_{e}$ :

$$
\left\{\begin{array}{l}
\left|Q T_{e}\left(e v_{1}, e v_{2}\right)\right|<25 \% M V \\
\dot{T}_{e}\left(e v_{1}, e v_{2}\right)<3 \mathrm{mV} / \mathrm{s}
\end{array}\right.
$$

- ERD, early repolarization duration;

- LRD, late repolarization duration;

- TRD, total repolarization duration.

ERD, $L R D$ and TRD are described in Fig. 2.

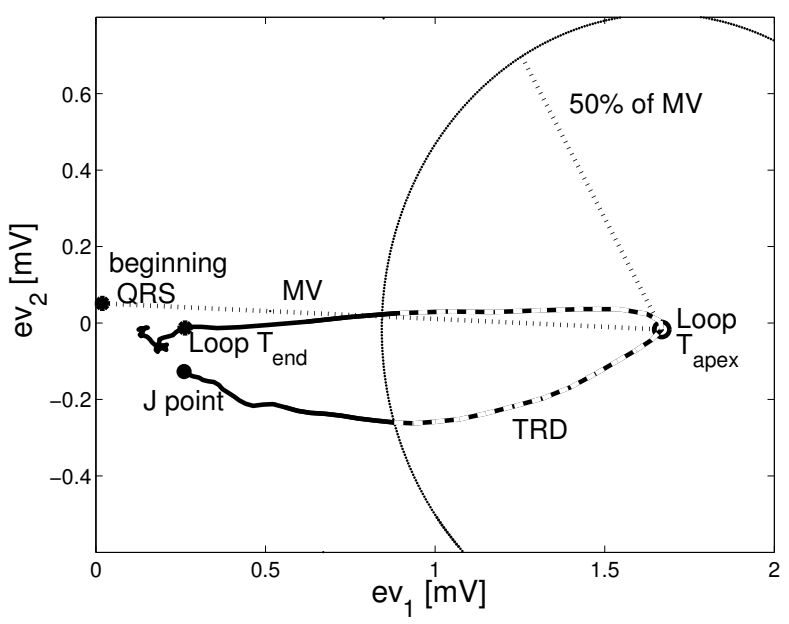

Figure 2. Definition of Maximum Vector (MV) in preferential plane and TRD.

From the position of the T-loop coinciding with MV, a circle of radius $(R=50 \% M V)$ is computed (Fig. 2); 
ERD is defined as the duration in $m s$ from the first sample the loop enters the $50 \%$ circle to the sample locating the MV position. Similarly, LRD is the duration from the time coinciding with MV position to the time when the loop first exits the circle; while $T R D=E R D+L R D$.

These durations do not include the QRS complex, but focus on the T-wave when the repolarization process reflects a large number of repolarizing cells.

\section{Results}

We reported the difference of measurement values at the same period of time between the two days: drug day and baseline day. Thus, we minimize potential effects of the autonomic nervous regulation of the heart.

In our preliminary analysis, we compared QT and TRV for the same heart rate. The differences between the first and the second day were computed within each patient, and these differences were average on the overall population. Table 1 shows the results for HR ranging from 57 to $60 \mathrm{bpm}$.

The values from the first column are the differences found between the first day and the second day before the drug was given to subjects ( $1^{\text {st }}$ hour of the recording). TRV is significantly increased 1 hour after dosing, while the QT interval remains in a normal range. After 1 hour and 15 minutes after dosing the QT interval becomes significantly prolonged $(24.6 \mathrm{~ms}, p<0.05)$. These results reveal the presence of "notches" and higher frequencies components inside the T-wave.

Table 1. Difference between on-drug and off-drug periods for QT and TRV indices.

\begin{tabular}{c||c|c|c|c}
$\begin{array}{c}\Delta \\
\begin{array}{c}\text { on drug - } \\
\text { baseline }\end{array}\end{array}$ & $\begin{array}{c}\text { baseline } \\
\text { before } \\
\text { dosing }\end{array}$ & $\begin{array}{c}15^{\prime}-45^{\prime} \\
\text { after } \\
\text { dosing }\end{array}$ & $\begin{array}{c}45^{\prime}-1 \mathrm{~h} 15^{\prime} \\
\text { after } \\
\text { dosing }\end{array}$ & $\begin{array}{c}1 \mathrm{~h} 15^{\prime}-2 \mathrm{~h} 15^{\prime} \\
\text { after } \\
\text { dosing }\end{array}$ \\
\hline \hline N. Subjects & 10 & 10 & 9 & 9 \\
\hline RR (ms) & -0.3 & -0.4 & 0.4 & 2.8 \\
\hline QT (ms) & -2.9 & -5.5 & 2.1 & $24.6^{*}$ \\
\hline TRV (\%) & -0.95 & $9.02^{*}$ & $6.39^{*}$ & $9.25^{*}$
\end{tabular}

$* p<0.05$; nonparametric test, comparison to baseline.

Sotalol slows the cardiac rhythm and thus a comparison of repolarization measurements at same heart rate becomes difficult to realize. Thus, several parameters were corrected for HR based on individual correction [10] using 6 hours baseline.

The differences in repolarization measurements, in reference to baseline, were not significantly different from 0 during the first $42 \mathrm{~min}$ after dosing (Table 2). Within the interval 42' - 48' after dosing $L S T_{c}$ becomes significant, showing that sotalol affects the ascending part of the Twave. This happens prior to significant QT prolongation: $Q T_{c}$ LII becomes significant only 1 hour after dosing. Un-
Table 2. Values of repolarization indices at different time intervals. Values are the difference between baseline and on-drug (for 6 minutes intervals).

\begin{tabular}{c||c|c|c|c}
$\begin{array}{c}\Delta \\
\begin{array}{c}\text { on drug - } \\
\text { baseline }\end{array}\end{array}$ & $\begin{array}{c}42^{\prime}-48^{\prime} \\
\text { after } \\
\text { dosing }\end{array}$ & $\begin{array}{c}48^{\prime}-54^{\prime} \\
\text { after } \\
\text { dosing }\end{array}$ & $\begin{array}{c}54^{\prime}-1 \mathrm{~h} \\
\text { after } \\
\text { dosing }\end{array}$ & $\begin{array}{c}1 \mathrm{~h}-1 \mathrm{~h} \mathrm{6} \\
\text { after } \\
\text { dosing }\end{array}$ \\
\hline \hline$Q T_{c} L I I(\mathrm{~ms})$ & $4.0(16)$ & $4.1(16)$ & $4.3(16)^{*}$ & $13(17)^{*}$ \\
\hline$L o o p Q T_{c}(\mathrm{~ms})$ & $2.3(13)$ & $2.8(14)$ & $4.2(14)$ & $8.1(15)^{*}$ \\
\hline$L S T_{c}$ & -1.07 & -1.07 & -0.97 & -1.98 \\
$(\mu V / m s)$ & $(3.08)^{*}$ & $(2.62)^{*}$ & $(2.43)^{*}$ & $(3.24)^{*}$ \\
\hline$E R D_{c}(\mathrm{~ms})$ & $5.1(14)^{*}$ & $4.9(14)^{*}$ & $4.4(12)$ & $9.5(16) \dagger$ \\
\hline$L R D_{c}(\mathrm{~ms})$ & $1.1(6.2)^{*}$ & $0.6(5.2)^{*}$ & $0.3(6.5)$ & $4.0(5.9) \dagger$ \\
\hline$T R D_{c}(\mathrm{~ms})$ & $5.7(19)$ & $5.9(18)$ & $2.9(16)^{*}$ & $11.0(20)^{*}$
\end{tabular}

$* p<0.05 ; \dagger p \leq 0.002$ nonparametric test, in comparison to baseline. Median values and Std between subjects in parenthesis.

der the effect of drug, T-waves are flatter with a lower left slope.

$E R D_{c}$ and $L R D_{c}$ evidence an effect of sotalol on the morphology of the T-wave prior to QT prolongation. One hour after dosing, all parameters appear to be significantly modified.

\section{Discussion and conclusions}

We demonstrated significant drug-induced repolarization abnormalities, corresponding to slower velocity and perturbed ascending T-wave. Abnormalities were present throughout the overall repolarization process but they were more pronounced in the ascending T-wave. The descending part of the T-wave $\left(T_{\text {peak }} T_{\text {end }}\right)$ was less affected and only at later time after dosing. In Fig. 3 an example of drug-induced perturbed T-loop is presented on its preferential plane.

We believe that the repolarization abnormalities we observed are strictly associated with the change of the delayed rectifier potassium current of the myocardium cells achieved by sotalol.

It is the efflux of potassium ions that produces the Twave of the electrocardiogram. Agents that delay or prevent this movement of potassium will modify the appearance of the T-wave. More precisely, $I_{K r}$ is a current moving potassium ions out of the cardiac cell during the plateau phase of the action potential. One may expect to see changes on the surface ECG recordings in the early phase of the T-wave as well as at the end of the wave. Our results are supporting such hypothesis.

Also the later part of the T-wave seems to be modified, being consistence with other studies in which $T_{\text {peak }} T_{\text {end }}$ was increased [11].

We suppose that sotalol affects differently the permeability to potassium ions in the different layer of the myocardium. The effect on $I_{K r}$ is therefore heterogeneous in the myocardium, resulting in a perturbed and heterogenous 

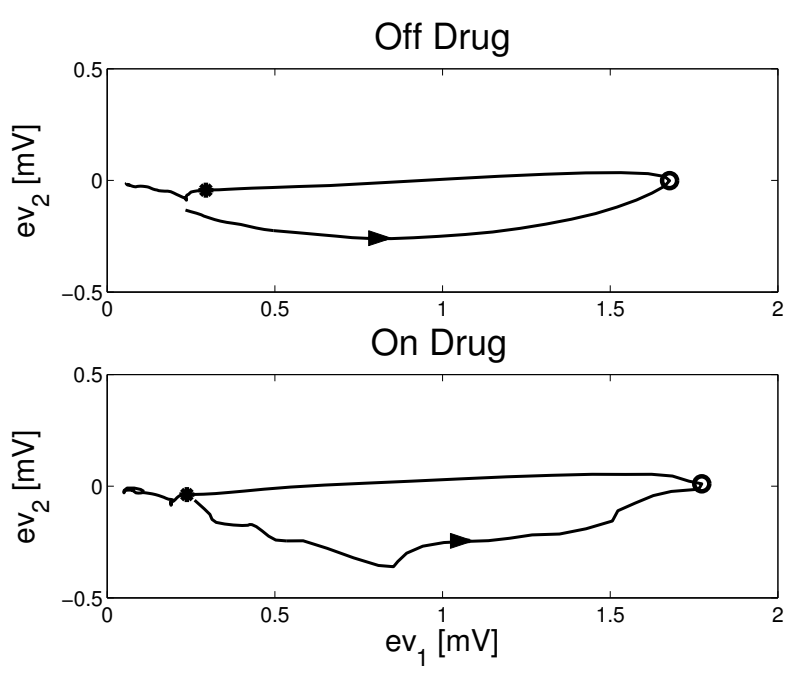

Figure 3. Comparison of T-loop of two cardiac beats from the same patient, with same HR and almost same QT, baseline vs after dosing, where fractionated repolarization is present.

repolarization. The effects on the surface ECG are lower and more fractionated T-waves.

T-wave abnormalities have been identified on $L Q T_{2}$ patients with HERG mutations [12]. In this type of patients, the $I_{K r}$ currents are diminished due to ion dysfunction. This leads to profound modifications to the duration and shape of the action potential. On the surface ECG the phenotopic expression of the mutation is shown as a reduction of the $\mathrm{T}$ wave ascending slope and an increased QT interval duration. Sotalol mimics the effect of the HERG mutation on the monophasic action potential. Thus, one would expect to find similar T-wave abnormalities as in $L Q T_{2}$ patients. Our study revealed indeed that these abnormalities are similar and they strengthen our hypothetical association between $I_{K r}$ abnormalities and $\mathrm{T}$ wave morphologic changes.

In conclusion, analyzing repolarization morphology might help identifying early drug-induced repolarization changes, which could be missed when relying exclusively on QT measurements. In our experience, early repolarization phase seems to be particularly affected by low levels of the drug and our quantitative parameters are able to identify these changes prior to appearance of drug-induced QT prolongation.

\section{References}

[1] Couderc J, Zareba W, Moss A. Drug-induced changes of ventricular repolarization: New incentives for quantifying $\mathrm{T}$ wave morphology. Int $\mathrm{J}$ Biolectromagnetism 2003; 5(1):167-170.
[2] Sarapa N, Morganroth J, Couderc J, Francom S, Darpo B, Fleishaker C, McEnroe D, Chen W, Zareba W, Moss A. Electrocardiographic identification of drug-induced QT prolongation: assessment by different recording and measurement methods. ANE 2004;9:48-57.

[3] Jolliffe I. Principal Component Analysis. Second edition. Springer, 2002.

[4] Okin P, Devereux R, Fabsitz R, Lee E, Galloway J, Howard B. Principal component analysis of the $\mathrm{T}$ wave and prediction of cardiovascular mortality in american indians. Circulation 2002;105:714-719.

[5] Priori S, Mortara D, Napolitano C, Diehl L, Paganini V, Cant F, Cant G Schwartz P. Evaluation of the spatial aspects of T-wave complexity in the Long-QT syndrome. Circulation 1997;96:3006-3012.

[6] Zabel M, Malik M, Hnatkova K, Papademetriou V, Pittaras A, Fletcher R, Franz M. Analysis of T-wave morphology from the 12-lead electrocardiogram for prediction of longterm prognosis in male US veterans. Circulation 2002; 105:1066-1070.

[7] Zabel M, Acar B, Klingenheben T, Franz M, Hohnloser S, Malik M. Analysis of 12-lead T-wave morphology for risk stratification after myocardial infarction. Circulation 2000; 10(5):1252-1257.

[8] Press W, Flannery B, Teukolsky S, Vetterling W. Numerical Recipes in C : The Art of Scientific Computing. Second edition. Cambridge University Press, 1992.

[9] Couderc J, Zareba W, Moss A, Sarapa N, Morganroth J, Darpo B. Identification of sotalol-induced changes in repolarization with $\mathrm{T}$ wave area-based repolarization duration parameters. J Electrocardiology 2003;36:115-120.

[10] Couderc J, Xia X, Zareba W, Moss A. Assessment of the stability of the individual-based correction of QT interval for heart rate. Ann Noninvasive Electrocardiol Jan 2005; 10(1):25-34.

[11] Fish J, Di Diego J, Nesterenko V, Antzelevitch C. Epicardial activation of left ventricular wall prolongs QT interval and transmural dispersion of repolarization. Circulation 2004;109:2136-2142.

[12] Couderc J, Zareba W, Moss A. Discrimination of HERG carrier from non-carrier adult patients with borderline prolonged $Q T_{c}$ interval. Computers in Cardiology 2005; To Appear.

Address for correspondence:

Martino Vaglio

HRFUP Cardiology Department

601 Elmwood Ave, Box 653

Rochester NY 14642 USA

ph +15852733578

fax +15852735283

Martino.Vaglio@heart.rochester.edu 